\title{
Sirs-Si Model of Malaria Disease with Application of Vaccines, Anti-Malarial Drugs, and Spraying
}

\author{
Randita Gustian Putri, Jaharuddin, Toni Bakhtiar \\ (Department of Mathematics, Bogor Agricultural University, \\ Jl. Meranti, Kampus IPB Darmaga, Bogor 16680, Indonesia)
}

\begin{abstract}
Malaria is a deadly disease transmitted to humans through the bite of infected female mosquitoes. It can also be transmitted from an infected mother (congenitally) or through blood transfusion. In this paper, we discussed the transmission of malaria featuring in the framework of an SIRS-SI model with treatments are given to humans and mosquitoes. We here utilized the use of vaccines, the use of anti-malarial drugs, and the use of spraying as treatment efforts. A stability analysis was then performed and numerical simulation was provided to clarify the result. It is shown that treatments affect the dynamics of human and mosquito populations. In addition, we proposed the Homotopy Analysis Method (HAM) to construct the approximate solution of the model.
\end{abstract}

Keywords: HAM, Malaria model, SIRS-SI model, Stability analysis, Treatment

\section{Introduction}

Malaria is an infectious disease caused by a parasite known as plasmodium. Carrier of plasmodium parasite is the female anopheles mosquito that causes the destruction of red blood cells in humans and animals through bites. Malaria can be transmitted through blood transfusion, sharing needles, or congenital.

Many researchers have developed a mathematical model of malaria transmission. SIR with nonlinear infection rate and the use of vaccination against the human population was analyzed by [1]. In this model it was assumed that the vaccination at the right time can cause susceptible human beings vaccinated can move directly to recovered class. The effect of treatment as a control variable on malaria transmission system was studied in the framework of optimal control theory by [2]. Model of malaria transmission was investigated in [3] by considering the existence of human-to-human transmission through blood transfusions and through malariainfected pregnant women (congenital).

In this current work, we discuss the effectiveness of the use of drugs in a malaria transmission model based on [3]. Modification of the model is done by considering the assumption that humans belong to recovered class have possibility to be susceptible, i.e., we consider a SIRS-SI model [4]. Moreover, we also consider the application of vaccine and spraying as introduced in [5,6]. Stability analysis is then performed to reveal the effects of treatments on population dynamics. Additionally we also propose the use of the homotopy analysis method in providing an approximate solution of the model.

\section{Mathematical Model}

In constructing the model we employ the following assumptions. We assume that the human population is divided into three classes, namely susceptible human $S_{h}$, infected human $I_{h}$, and recovered human $R_{h}$, while the mosquito population is grouped into two classes: susceptible mosquito $S_{m}$ and infected mosquito $I_{m}$. Individuals who are born and migrate to the susceptible class has a constant rate of $\lambda_{h}$. Humans in susceptible class can move into the infected class due to blood transfusion at a rate of $a \beta_{1}$ (with $a$ is average number of blood transfusion per unit time and $\beta_{1}$ is the chances of disease transmission to susceptible human from infected human) or by an infected mosquito bite at a rate of $b \beta_{2}$ (with $b$ is average number of infected mosquito bites on susceptible human per unit time and $\beta_{2}$ is the chances of disease transmission from infected mosquitoes to susceptible humans). Humans in susceptible class can move into the recovered class due to vaccination at a rate of $\theta$. Humans insusceptible class may die at a rate of $\mu_{h}$. A newborn baby can be infected malaria due to congenital with a rate of $\eta$. Humans in infected class can move to the recovered class due to the use of antimalarial drugs with a rate of $k \gamma$ (with $k$ is the rate of human recovery and $\gamma$ is the effectiveness of anti-malarial drugs). Humans in infected class can die at a rate of $\mu_{h}$ and death due to malaria at a rate of $\alpha$. Humans in recovered class $\left(R_{h}\right)$ can die at a rate of $\mu_{h}$.

Furthermore, mosquitoes are born and migrate to susceptible class with a constant rate of $\lambda_{m}$. Mosquitoes in susceptible class may move into the infected class for biting infected humans at a rate of $c \beta_{3}$ (with $c$ is average number of susceptible mosquito bites on infected humans per unit time and $\beta_{3}$ is the chances of disease transmission from infected humans to susceptible mosquitoes) or can die at a rate $\mu_{m}$. Mosquitoes in susceptible class and infected class can die because the use of spraying at a rate of $\rho$. Mosquitoes 
in infected class can die at a rate $\mu_{m}$.Compartmental diagram of the model is illustrated in Fig.1 and its dynamical equations are formulated by system (1) as follows:

$$
\begin{aligned}
& \frac{d S_{h}}{d t}=\lambda_{h}+\sigma R_{h}-\left(a \beta_{1} I_{h}+b \beta_{2} I_{m}\right) S_{h}-\left(\theta+\mu_{h}\right) S_{h}, \\
& \frac{d I_{h}}{d t}=\eta I_{h}+\left(a \beta_{1} I_{h}+b \beta_{2} I_{m}\right) S_{h}-\left(\mu_{h}+\alpha+k \gamma\right) I_{h}, \\
& \frac{d R_{h}}{d t}=k \gamma I_{h}-\left(\mu_{h}+\sigma\right) R_{h}+\theta S_{h}, \\
& \frac{d S_{m}}{d t}=\lambda_{m}-\left(c \beta_{3} I_{h}+\mu_{m}+\rho\right) S_{m}, \\
& \frac{d I_{m}}{d t}=c \beta_{3} I_{h} S_{m}-\left(\mu_{m}+\rho\right) I_{m} .
\end{aligned}
$$

\section{Stability Analysis}

\section{A. Equilibrium State}

Equilibrium points can be obtained by simultaneously solving the following equations:

$$
\frac{d S_{h}}{d t}=\frac{d I_{h}}{d t}=\frac{d R_{h}}{d t}=\frac{d S_{m}}{d t}=\frac{d I_{m}}{d t}=0 .
$$

System (1) has two types of equilibrium points, namely disease-free equilibrium point $x_{d f e}$ and endemic equilibrium point $x_{e e}$. It is easy to verify that

where

$$
x_{d f e}\left(S_{h}, I_{h}, R_{h}, S_{m}, I_{m}\right)=\left(S_{h}^{*}, 0, R_{h}^{*}, S_{m}^{*}, 0\right),
$$

and

$$
S_{h}^{*}=\frac{\lambda_{h}\left(\mu_{h}+\sigma\right)}{\mu_{h}\left(\theta+\sigma+\mu_{h}\right)}, R_{h}^{*}=\frac{\lambda_{h} \theta}{\mu_{h}\left(\theta+\sigma+\mu_{h}\right)}, S_{m}^{*}=\frac{\lambda_{m}}{\mu_{m}+\rho},
$$

where

$$
x_{e e}\left(S_{h}, I_{h}, R_{h}, S_{m}, I_{m}\right)=\left(S_{h}^{* *}, I_{h}^{* *}, R_{h}^{* *}, S_{m}^{* *}, I_{m}^{* *}\right),
$$

$$
\begin{gathered}
S_{h}^{* *}=\frac{\lambda_{h}+\sigma R_{h}^{* *}}{a \beta_{1} I_{h}^{* *}+b \beta_{2} I_{m}^{* *}+\theta+\mu_{h}}, I_{h}^{* *}=\frac{b \beta_{2} I_{m}^{* *} S_{h}^{* *}}{\mu_{h}+k \gamma+\alpha-a \beta_{1} S_{h}^{* *}-\eta}, R_{h}^{* *}=\frac{\theta S_{h}^{* *}+k \gamma I_{h}^{* *}}{\mu_{h}+\sigma}, \\
S_{m}^{* *}=\frac{\lambda_{m}}{c \beta_{3} I_{h}^{* *}+\mu_{m}+\rho}, I_{m}^{* *}=\frac{c \beta_{3} I_{h}^{* *} S_{m}^{* *}}{\mu_{m}+\rho} .
\end{gathered}
$$

\section{B. Basic Reproduction Number}

Basic reproductive number $R_{0}$ is denoted by the expectation value of the number of infections per unit time. This infection occurs in a susceptible population produced by one infected individual.To determine the basic reproduction number we use the next generation matrix approach [7]. Based on system (1), we may define matrices $F$ and $V$ as follow

$$
F=\left(\begin{array}{cc}
\frac{a \beta_{1} \lambda_{h}\left(\mu_{h}+\sigma\right)}{\mu_{h}\left(\theta+\sigma+\mu_{h}\right)} & \frac{b \beta_{2} \lambda_{h}\left(\mu_{h}+\sigma\right)}{\mu_{h}\left(\theta+\sigma+\mu_{h}\right)} \\
\frac{c \beta_{3} \lambda_{m}}{\mu_{m}+\rho} & 0
\end{array}\right), V=\left(\begin{array}{cc}
-\eta+\mu_{h}+\alpha+k \gamma & 0 \\
0 & \mu_{m}+\rho
\end{array}\right) .
$$

Basic reproductive number $R_{0}$ is the largest positive eigen values of the matrix $K=F V^{-1}$, from which we obtain

where

$$
R_{0}=\frac{b_{1}+\sqrt{b_{1}^{2}+4 b_{2} b_{3}}}{2}
$$

$$
\begin{gathered}
b_{1}=\frac{a \beta_{1} \lambda_{h}\left(\mu_{h}+\sigma\right)}{\mu_{h}\left(\theta+\sigma+\mu_{h}\right)\left(-\eta+\mu_{h}+\alpha+k \gamma\right)}, b_{2}=\frac{b \beta_{2} \lambda_{h}\left(\mu_{h}+\sigma\right)}{\left(\mu_{m}+\rho\right) \mu_{h}\left(\theta+\sigma+\mu_{h} \lambda_{m}\right.}, \\
b_{3}=\frac{}{\left(\mu_{m}+\rho\right)\left(-\eta+\mu_{h}+\alpha+k \gamma\right)} .
\end{gathered}
$$

According to the basic reproduction number we say that: if $R_{0}<1$ then the number of infected individuals will decrease with each generation, so that the disease will not spread, and if $R_{0}>1$ then the number of infected individuals will increase with each generation, so that the disease will spread. 


\section{Simulation}

In this simulation, the population dynamics are observed in conditions such that $R_{0}<1$. Thus we would like to show the effect of vaccination, anti-malarial drug, and spraying in a situation where the disease doesn't spread. The selection of parameters is relied on the studies conducted by various reliable sources. Based on [2] we use the following values $\mu_{h}=0.0004, \mu_{m}=0.04, \sigma=1 / 730, \alpha=0.05$. According to [8] we have: $\lambda_{h}=0.027, b=0.13, \beta_{2}=0.010, \beta_{3}=0.072$, and from [1] we have $k=0.611$. The following parameter values are assumed based on the most common situation $\lambda_{m}=0.13, a=0.038, c=0.022, \beta_{1}=0.02, \rho \in$ $[0,1], \theta \in[0,1], \eta=0.005, \gamma \in[0.01,1], S_{h}(0)=40, I_{h}(0)=2, R_{h}(0)=0, S_{m}(0)=500, I_{m}(0)=10$.

\section{1) Simulation of the effectiveness of the use anti-malarial drugs}

Simulation is performed to demonstrate the effectiveness of the use of anti-malaria drugs on human populations and mosquito populations. In this case, it will be shown that an increase or decrease in the value of the parameter $\gamma$ can alter the basic reproduction number $R_{0}$ defined in (4). The change in value of $\gamma$ and thus $R_{0}$ can be seen in Table 1. In the human population, as shown in Fig.2, if the effectiveness of the use of antimalarial drugs is increased, then it increases the number of susceptible humans as well as the number of recovered humans, but it decreases the number of infected humans. The use of anti-malarial drugs given to human has also an impact on the mosquito population, as shown in Fig.3. The same treatment on $\gamma$ causes a decrease in the number of infected mosquitoes but an increase in the number of susceptible mosquitoes. Increasing or decreasing in the number of humans and mosquitoes in each class tend to be equal to any increase in the effectiveness of the use of anti-malarial drugs. The maximum number of infected humans and mosquitoes occurs at $t=25$ days. At this point, the effectiveness level of $20 \%$ can reduce the number of infected humans up to $23.81 \%$ of the total human population and can reduce the number of infected mosquitoes up to $5.88 \%$.

\section{2) Simulation of the effectiveness of the use vaccine}

In this part we demonstrate the effectiveness of the use of vaccines on human and mosquito populations. It is assumed that infected humans treated with anti-malarial drugs by $10 \%$. In this case, it will be shown the effect of parameters $\theta$ and the basic reproduction number $R_{0}$ to the population dynamics. The change in value of the parameters $\theta$ and $R_{0}$ can be seen in Table 2. In the human population, as shown in Fig.4, if the effectiveness of the vaccine is improved and the other parameters are fixed, then the number of humans in susceptible and recovered classes are increased. It indirectly causes a decrease in the number of human in infected class. While, in the mosquito population as shown in Fig.5, improvement on vaccine effectiveness indirectly decreases the number of mosquito in infected class.

\section{3) Simulation of the use effectiveness of the use spraying}

Now we demonstrate the effectiveness of the use of spraying on human populations and mosquito populations. In this case we still assumed that infected humans consume anti-malarial drugs by $10 \%$ and we show the effect of parameter $\rho$ and $R_{0}$ on population dynamics. The change in value of the parameter $\rho$ and $R_{0}$ can be seen in Table 3. In Fig.6 we can see that an improvement on the effectiveness of spraying increases the number of susceptible humans, but decreases the numbers of infected and recovered humans. Meanwhile, toward the vector population, the same treatment decreases the population in both classes as spraying is aimed to mosquitoes.

\section{Application Of The Homotopy Analysis Method}

Based on [9], this section discusses the utilization of the so-called homotopy analysis method to derive an approximate solution of the SIRS-SI model of malaria disease. With respect to system (1), we define the following linear operators $\mathcal{L}_{i}(i=1,2,3,4,5)$ and nonlinear operators $\mathcal{N}_{i}(i=1,2,3,4,5)$ :

$$
\begin{aligned}
& \mathcal{L}_{i}[\bar{\phi}(t ; q)]=\frac{d \phi_{i}(t ; q)}{d t}, i=1,2,3,4,5 \\
& \mathcal{N}_{1}(\bar{\phi})=\frac{d \phi_{1}}{d t}-\lambda_{h}-\sigma \phi_{3}+\left(a \beta_{1} \phi_{2}+b \beta_{2} \phi_{5}\right) \phi_{1}+\left(\theta+\mu_{h}\right) \phi_{1} \\
& \mathcal{N}_{2}(\bar{\phi})=\frac{d \phi_{2}}{d t}-\eta \phi_{2}-\left(a \beta_{1} \phi_{2}+b \beta_{2} \phi_{5}\right) \phi_{1}+\left(\mu_{h}+k \gamma+\alpha\right) \phi_{2} \\
& \mathcal{N}_{3}(\bar{\phi})=\frac{d \phi_{3}}{d t}-k \gamma \phi_{2}+\left(\mu_{h}+\sigma\right) \phi_{3}-\theta \phi_{1} \\
& \mathcal{N}_{4}(\bar{\phi})=\frac{d \phi_{4}}{d t}-\lambda_{m}+\left(c \beta_{3} \phi_{2}+\mu_{m}+\rho\right) \phi_{4} \\
& \mathcal{N}_{5}(\bar{\phi})=\frac{d \phi_{5}}{d t}-c \beta_{3} \phi_{2} \phi_{4}+\left(\mu_{m}+\rho\right) \phi_{5} .
\end{aligned}
$$


In (5) $q \in[0,1]$ is a parameter and $\bar{\phi}=\left(\phi_{1}, \phi_{2}, \phi_{3}, \phi_{4}, \phi_{5}\right)$ is a function that depends on $t$ and $q$. Based on (5) and (6) we then construct zero-order deformations as follow:

$$
(1-q) \mathcal{L}_{i}\left[\phi_{i}(t, q)-\phi_{i, 0}(t)\right]=q h \mathcal{N}_{i}\left[\phi_{i}(t, q)\right], i=1,2,3,4,5 \text {. }
$$

with $h$ is an auxiliary variable. In (7), if $q=0$ and $q=1$, then from (7) we have $\phi_{i}(t, 0)=\phi_{i, 0}(t)$ and $\phi_{i}(t, 1)=\phi_{i}(t)$. Using the concept of Taylor series, $\phi_{i}(t ; q), i=1,2,3,4,5$, can be decomposed into

where

$$
\phi_{i}(t, q)=\phi_{i, 0}(t)+\sum_{n=1}^{\infty} \phi_{i, n}(t) q^{n}
$$

$$
\phi_{i, n}(t)=\left.\frac{1}{n !} \frac{d^{n} \phi_{i}(t, q)}{d q^{n}}\right|_{q=0}
$$

If $q=1$, equation (8) becomes

$$
\phi_{i}(t, 1)=\phi_{i, 0}(t)+\sum_{n=1}^{\infty} \phi_{i, n}(t), i=1,2,3,4,5 .
$$

Next, for $i=1,2,3,4,5$, set the $n$-th order equations as follows

where

$$
\mathcal{L}\left[\phi_{i, n}(t)-\chi_{n} \phi_{i, n-1}(t)\right]=h R_{i, n}\left(\bar{\phi}_{i, n-1}\right), i=1,2,3,4,5,
$$

$$
\begin{aligned}
& R_{1, n}=\frac{d}{d t} \phi_{1, n-1}-\lambda_{h}\left(1-\chi_{n}\right)-\sigma \phi_{3, n-1}+a \beta_{1} \sum_{k=0}^{n-1} \phi_{1, k} \phi_{2, n-1-k}+b \beta_{2} \sum_{k=0}^{n-1} \phi_{1, k} \phi_{5, n-1-k}+\left(\theta+\mu_{h}\right) \phi_{1, n-1}, \\
& R_{2, n}=\frac{d}{d t} \phi_{2, n-1}-\eta \phi_{2, n-1}-a \beta_{1} \sum_{k=0}^{n-1} \phi_{1, k} \phi_{2, n-1-k}-b \beta_{2} \sum_{k=0}^{n-1} \phi_{1, k} \phi_{5, n-1-k}+\left(\mu_{h}+k \gamma\right) \phi_{2, n-1}, \\
& R_{3, n}=\frac{d}{d t} \phi_{3, n-1}-k \gamma \phi_{2, n-1}+\left(\mu_{h}+\sigma\right) \phi_{3, n-1}-\theta \phi_{1, n-1}, \\
& R_{4, n}=\frac{d}{d t} \phi_{4, n-1}-\lambda_{m}\left(1-\chi_{n}\right)+c \beta_{3} \sum_{k=0}^{n-1} \phi_{4, k} \phi_{2, n-1-k}+\left(\mu_{m}+\rho\right) \phi_{4, n-1}, \\
& R_{5, n}=\frac{d}{d t} \phi_{5, n-1}-c \beta_{3} \sum_{k=0}^{n-1} \phi_{4, k} \phi_{2, n-1-k}+\left(\mu_{m}+\rho\right) \phi_{5, n-1},
\end{aligned}
$$

with $\chi_{n}=0$ for $n \leq 1$ and $\chi_{n}=1$ for $n>1$.

We also employ the following initial values: $\phi_{1,0}(t)=S_{h}(0)=40, \phi_{2,0}(t)=I_{h}(0)=2, \phi_{3,0}(t)=R_{h}(0)=$ $0, \phi_{4,0}(t)=S_{m}(0)=500, \phi_{5,0}(t)=I_{m}(0)=10$.

The solution to the $n$-th order of $(8)$ is

$$
\phi_{i, n}(t)=\chi_{n} \phi_{i, n-1}(t)+h \int R_{i, n}\left(\bar{\phi}_{i, n-1}\right) .
$$

Thus, the approximate solution of the model up to the order of $N$ is expressed as follows

$$
\phi_{i}(t)=\sum_{n=0}^{N} \phi_{i, n}(t) \text {. }
$$

Therefore an approximate solution of system (1) can be derived by homotopy analysis method according to the following procedures:

1. For a given model represented by a system of differential equations, define a series of linear operators (5) and nonlinear operators (6).

2. Determine a set of $n$-th order equations given in (9) including their initial values and then solve (10).

3. Construct an approximate solution $\phi_{i}$ according to (11) up to order $N$.

From (10) and (11), the approximate solution obtained by homotopy analysis method depends not only on a time variable $t$ but also on an auxiliary variable $h$. The later variable should be selected in order to provide a proper approximate solution. The selection of auxiliary variables $h$ obtained by homotopy solution twice lowered to $q$ then evaluated at $q=0$. Curves intersecting $h$ interval would be the value of $h$ is taken in the solution of homotopy method. Based on Fig. 8 , the fifth curve is tangent to $h$ interval $-1.8 \leq h \leq 0.3$. Based on this interval, a value of $h$ can be chosen in order to obtain a solution with the absolute error is small when compared with a numerical solution. In this case, is chosen $h=-1$. With the selection of $h=-1$, then the homotopy solution will be obtained as a $t$ function. Fig.9 shows that the solution of homotopy analysis method (HAM) and numerical solution (NUM) Runge-Kutta up to 10-th order which has a small absolute error. Seen at a distance of two curves are quite close to solution. This means that the homotopy method can be used to solve SIRS-SI model of malaria disease. 


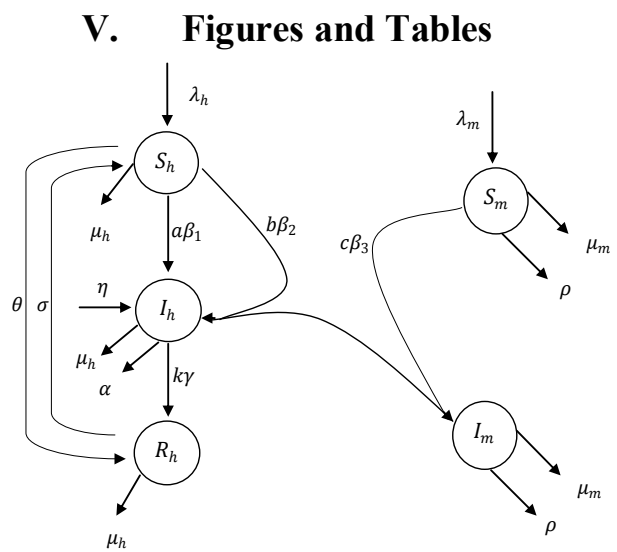

Human

Mosquito

Fig. 1: Compartmental diagram of malaria disease
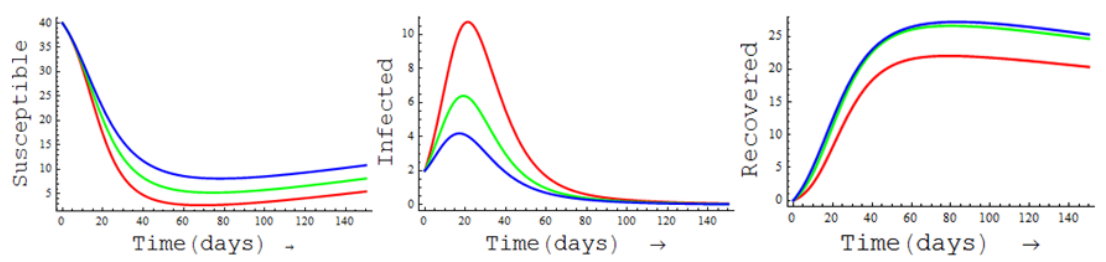

$-\gamma=0.05-\gamma=0.15-\gamma=0.25$

Fig.2: Dynamics of human population under the treatment of anti-malarial drugs

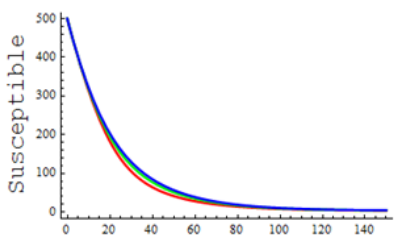

Time (days) $\rightarrow$

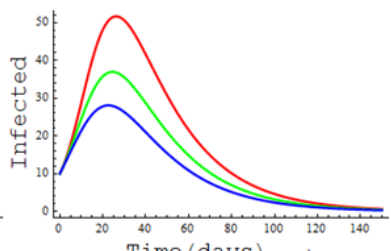

$-\gamma=0.05-\gamma=0.15-\gamma=0.25$

Fig.3: Dynamics of mosquito population under the treatment of anti-malarial drugs
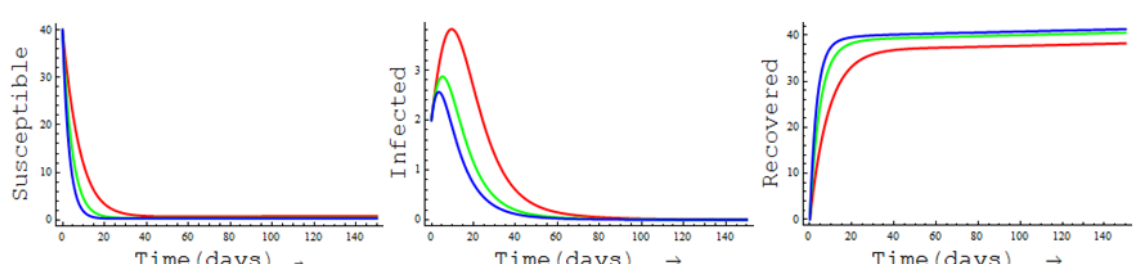

Time (days)

Time (days)

Time (days) $\rightarrow$

$$
-\theta=0.05-\theta=0.15-\theta=0.25
$$

Fig.4: Dynamics of human population under the treatment of vaccines

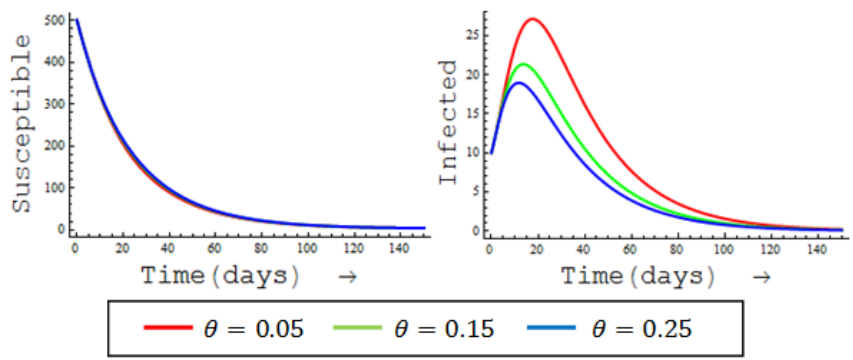

Fig.5: Dynamics of mosquito population under the treatment of vaccines 

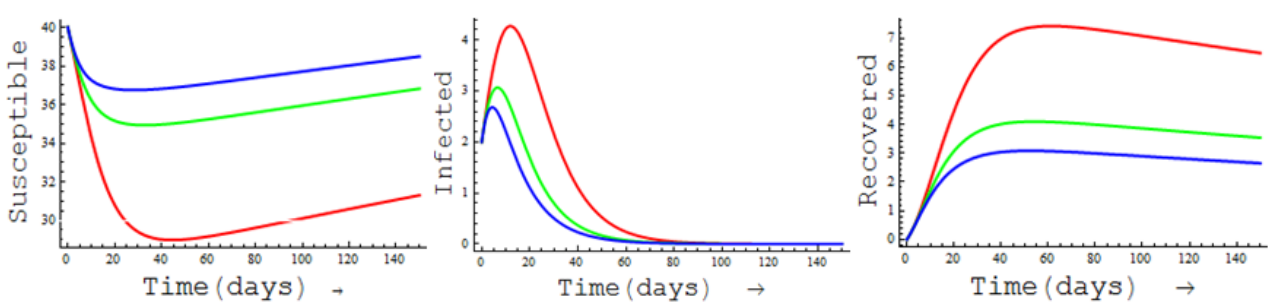

$$
-\rho=0.05-\rho=0.15-\rho=0.25
$$

Fig.6: Dynamics human population under the treatment of spraying

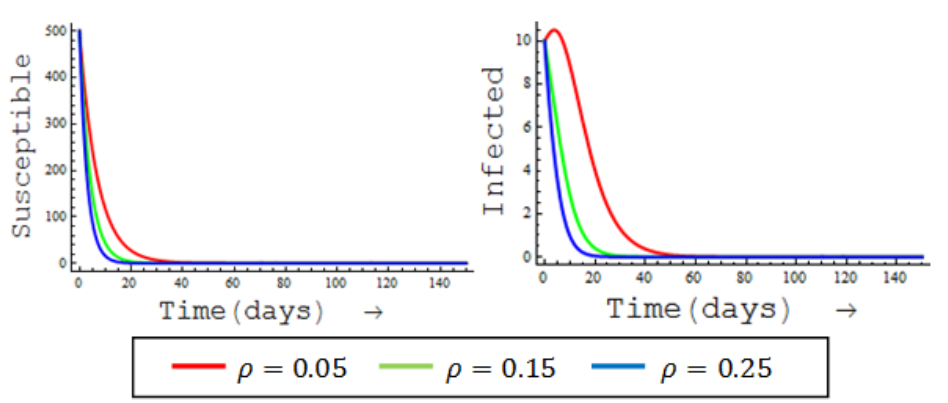

Fig.7: Dynamics of mosquito population under the treatment of spraying

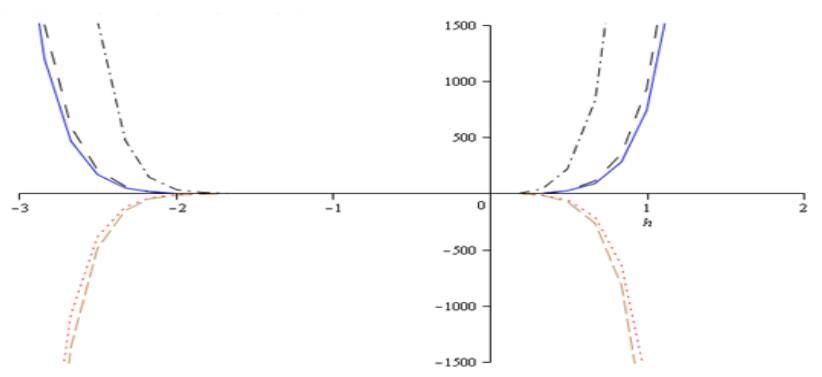

Fig.8: The $h$-curve up to 10 -th order

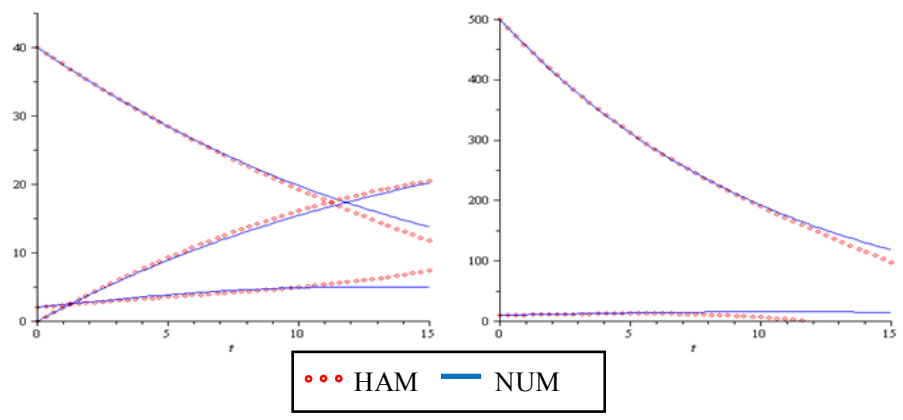

Fig.9: The solution of homotopy analysis method up to 10 -th order 
Table 1: Parameter values used for simulation of the effectiveness of antimalarial drugs

\begin{tabular}{|c|c|}
\hline Parameter $\gamma$ & Basic reproduction number \\
\hline$\gamma=0.10$ & $R_{0}=0.646$ \\
\hline$\gamma=0.20$ & $R_{0}=0.454$ \\
\hline$\gamma=0.30$ & $R_{0}=0.361$ \\
\hline
\end{tabular}

Table 2: Parameter values used for simulation of the effectiveness of vaccines

\begin{tabular}{|c|c|}
\hline Parameter $\theta$ & Basic reproduction number \\
\hline$\theta=0.10$ & $R_{0}=0.047$ \\
\hline$\theta=0.20$ & $R_{0}=0.039$ \\
\hline$\theta=0.230$ & $R_{0}=0.027$ \\
\hline
\end{tabular}

Table 3: Parameter values used for simulation of the effectiveness of spraying

\begin{tabular}{|c|c|}
\hline Parameter $\rho$ & Basic reproduction number \\
\hline$\rho=0.10$ & $R_{0}=0.499$ \\
\hline$\rho=0.20$ & $R_{0}=0.488$ \\
\hline$\rho=0.30$ & $R_{0}=0.485$ \\
\hline
\end{tabular}

\section{Conclusion}

We have presented an SIRS-SI model of malaria transmission equipped with a series of treatments namely the use of vaccines, anti-malarial drugs and spraying. Toward disease-free and endemic equilibrium points, we perform a stability analysis characterized by a basic reproduction number. Through a series of simulations we have shown the effects of treatments with different levels of effectiveness to human and mosquito populations. We have also proposed the use of so-called homotopy analysis method as an alternate technique in deriving an approximate solution of the model. It has been shown that such method can approximate the numerical solution quite well.

\section{References}

[1] Laarabi H, Labriji EH, Rachik M, Kaddar A. 2012. Optimal control of an epidemic model with a saturated incidence rate. Modelling and Control. Vol.17, No.4, pp. 448-459.

[2] Agusto FB, Marcus N, Okosun KO. 2012. Application of optimal control to the epidemiology of malaria. Electronic Journal of Differential Equation. Vol. 2012, No.81, pp. 1-22.

[3] Abdullahi MB, Hasan YA, Abdullah FA. 2013. A mathematical model of malaria and the effectiveness of drugs. Applied Mathematical Sciences. Vol. 7, 2013, No. 62, pp. 3079-3095.

[4] Mandal S, Sarkar RR, Sinha S. 2011. Mathematical models of malaria - a review. Malaria Journal. Vol. 10, pp. 1-19.

[5] Schwartz L, Brown GV, Genton B, Moorthy VS. 2012. A review malaria vaccine clinical projects based on the rainbow table. Malaria Journal. 11:11

[6] Ratovonjato et al. 2014. Entomological and parasitological impacts of indoor residual spraying with DDT, alphacypermethrin and deltamethrin in the western foothill area of Madagascar. Malaria Journal. 13:21

[7] Van Den Driessche P, Watmough J. 2008. Chapter 6: Further Notes on the Basic Reproduction Number. In: Brauer F, Van Den Driessche P, Wu, J. Mathematical Epidemiology. 1945. Lecture Notes in Mathematics, Springer, pp. 159-178.

[8] Chitnis NR. 2005. Using mathematical models in controlling the spread of malaria [dissertation]. Arizona (US): The University of Arizona.

[9] Liao. 2004. Beyond Perturbation: Introduction to the homotopy analysis method. New York (US): Boca Raton. 